\title{
Waiting lists for health care in Canada
}

W aiting lists for surgery get a lot of newspaper coverage nowadays. The idea that one has to wait for care is well established and often a reason for hand wringing, but it is mostly a myth. In Canada, nobody waits for care for severe injuries or the treatment of heart attacks; what they wait for is care they want when they want it, or essential care such as a surgical booking on very short notice with the heart surgeon with the highest profile. We tend to complain loudly that the government should fix whatever the latest problem is, and forget the complaint just as quickly. It is the same when patients demand an immediate appointment. Patients demand, but once they are seen, forget until the next time. We forget someone has to fix problems. We often look to others to fix our problems, perceived or real. Why is this? Is it our history of looking to monopoly to solve problems? Is it our basic distrust of initiative?

So what is wrong with waiting lists anyway? Nothing! All you have to do is plan ahead so the time of treatment is what you wanted anyway, or heaven forbid, get yourself on more than one waiting list, as long as there are competing lists. The problem with monopoly centres of excellence is that there is only one list.

It is assumed that those who know the most about health care (the doctors) will change the system for the better, so they themselves will not have to wait, thereby making things better for all. I don't think so; doctors assume (but never discuss) that one advantage of being on the inside is to work the system for yourself or your family. We have all done it.

The system has taboos, as all belief systems have, and one taboo is discussing why we have waiting lists. It can be difficult to discuss the beliefs we all live with, so we seldom do, but let me try.

There seem to be two basic systems available to supply any service or good.

One is the demand system, which is used by the shopkeeper who stocks a store and decides every day which item is going to move off the shelves. The shopkeeper has to take risks to stay in business. Some things he stocks are winners, and some are losers and are quickly taken off the shelves. He competes to provide the best service. If he chooses wrong too often, he goes out of business. The reward is not just income, which is taxed anyway; the reward is satisfaction in predicting the market.

Then there is the supply system. This system is controlled from the centre where the money comes from. The great advantage of the supply system is that the centre has the power to decide how much is spent on what. Its reason to exist is to control what is spent. The centre predicts what services will be needed many years into the future, and makes the decisions. This is the system most able to produce social change, because whatever group that can influence the centre makes the social change happen, or at least gets to try their favorite ideas. This system needs a monopoly to operate. The problem is that this system has to simulate the market for services, and it has to be right, so it has to spend on systems and future planning. In the 1990s when the centre decided to reduce medical school enrollments, the doctor shortage was not appreciated for many years, and then it was hard to catch up.

All this talk of systems is irrelevant to the average Canadian, who has a crafty skeptic's view of theory. We all like this mythical Canadian. He is pragmatic and hardheaded. He has a healthy distrust of government but will take whatever is being supplied as long as there are no strings attached (who wouldn't?).

$\mathrm{Mr}$ and Ms average Canadian want good health care, and he or she will wait as long as everyone else is waiting too, but, no way anyone is going to bump the line. The politicians being crafty know they cannot allow anyone to wait for essential services beyond what is acceptable, and so are drawing up waiting time lists, to make sure that no one ever waits for heart or cancer treatment, or emergency care.

Those of us who provide less essential (although important) health care services will likely be faced with less availability as these priorities are introduced. We will be looked at closely, told to speed up our surgery and fit into the parameters of the staff's union hours of work and hospital budgets. We will admit almost no one, and our ability to do so will be reduced as postsurgical care for our patients loses priority.

Waiting lists do not matter for nonessential services, in fact, they are an essential part of the supply system.

Waiting lists are useful because they indicate the parts of the system that have to be expanded. Without lists, it would not be clear that more capacity is needed. Alternatively, when the waiting list is close to zero, it is an indication that capacity is in excess, because if capacity was reduced the lists would lengthen, and the system could be made less expensive (because it could be effectively shrunk).

Lists respond to patient complaints about long lists. If no one complains, it does not matter how long the lists are. If people do not complain due to a sense of community, 'because we are all in this together', there is no need to reduce waiting lists. Therefore, complaints are essential feedback devices.

Waiting lists are easy to manipulate; just get on the list early and cancel if you need to. (There are no penalties for cancelling.) Despite all the talk about waiting lists, Canadians need not be concerned.

There will never be waiting lists for essential services. If the average Canadian sees a problem that affects him or her, there will be a cry for a mixed demand system, and politicians cannot allow that to happen. 